\title{
Dynamics Characterization of a U-Shaped Micro-Resonator Portal Frame
}

\author{
Rodrigo Tumolin Rocha ${ }^{\circledR}$, Nouha Alcheikh ${ }^{\circledR}$, Fahimullah Khan ${ }^{\circledR}$, and Mohammad I. Younis, Member, IEEE
}

\begin{abstract}
We present a study of the natural (resonance) frequencies of a tunable in-plane Microelectromechanical portal frame (U-shape) under DC electrostatic loads. The structure is designed and fabricated to excite the in-plane anti-symmetric (sway, first) and the symmetric (second) modes. Experimental results show high tunability of the resonance frequencies of both modes due to the electrostatic forces. Finite element simulations show good agreement with the experimental measurements. Further simulations are presented for the tunability of the natural frequencies of other higher-order modes using various electrode configurations. Frequency-response curves are presented for pure AC actuation of the first two modes showing strong nonlinear softening behavior due to the geometric nonlinearities of the portal frame.

[2020-0269]
\end{abstract}

Index Terms-Experimental measurements, finite element method, high tunability, micro fabrication, nonlinear dynamics.

\section{INTRODUCTION}

A S SENSING and actuation technologies continue to evolve, there is critical need for the development of smaller and smarter devices capable of providing new capabilities and solutions. A central platform toward this is micro-electromechanical systems (MEMS), which have the advantages of low-cost fabrication, small size, fast response, low power consumption, and high sensitivity. MEMS have been used in several applications, such as gas-sensors [1], [2], mass detection [2], [3], pressure sensors [4], bioapplications [5], [6], electrothermal actuations [6], [7], energy harvesting [8], [9], Radio Frequency (RF) switches [10], [11], logics [12], [13], filters [14], and control [15].

In addition, MEMS are attractive due to their flexibility, reliability, feasibility, and diversity of applications. Thus, the study and optimization of MEMS devices become very important so that improved prototypes and even novel applications can be realized.

In macro-sized systems, it can be challenging to reveal the rich dynamic properties due to difficulties in controlling precisely and in a wide range the stiffness, damping, and forcing of the system. At the micro-scale, on the other hand, stiffness can be tuned through, for example, electrostatic and

Manuscript received July 13, 2020; revised August 12, 2020; accepted August 24, 2020. This work was supported by the King Abdullah University of Science and Technology (KAUST). Subject Editor N. Barniol. (Corresponding author: Mohammad I. Younis.)

The authors are with the Physical Sciences and Engineering Division, King Abdullah University of Science and Technology (KAUST), Thuwal 239556900, Saudi Arabia (e-mail: mohammad.younis@kaust.edu.sa).

Color versions of one or more of the figures in this article are available online at http://ieeexplore.ieee.org.

Digital Object Identifier 10.1109/JMEMS.2020.3020230 electrothermal actuation, damping can be controlled through environmental chambers and design variations, and forcing can be applied in a wide range through voltage changes. Hence, large deformations of microstructures can be induced amplifying the effect of their geometric nonlinearities. Such nonlinear behaviors have been cited in several studies [16]-[31]. Recent advances of linear and nonlinear dynamics of MEMS are surveyed in [32].

Nonlinearities in straight micro beams have been extensively studied the past two decades when they are excited near resonance conditions under electrostatic DC and AC voltages. Jump phenomenon is the most common, which prevails in beams due to, for example, mid-plane stretching in clamped-clamped beams, internal resonances between modes of vibrations, and softening effect of the electrostatic forces [33]-[36]. Jumps can be of softening or hardening types.

Simple structures, such as single beams, have been commonly employed in MEMS due to the ease of accessing their modes of vibration and exploring their linear and nonlinear dynamical behaviors. Compound and more complex structures have not been commonly used in dynamic-based applications. These structures have been more frequently implemented at the macro-scale.

Compound structures can have various forms, such as Lshape [37], [38], T-shape [39], and U-shape (portal frame) beams [40]-[46], which are commonly called frames, and triangular shapes, such as trusses. In micro-scale systems, such compound structures can be promising for various applications, such as energy harvesting, sensing, and actuation. Nevertheless, these structures have been reported mostly for static applications, and mainly in the linear regime, without considering or accounting properly for the effect of their nonlinearities [37]-[46].

Portal frames have been commonly employed and studied in macro structures in urban and domestic applications, for example in bridges and trusses. According to Brasil and Mazzilli [46], when these structures undergo large vibration, geometric nonlinearities are activated, and nonlinear behavior becomes important. Nonlinear behaviors in portal frames, such as the saturation phenomenon, have been reported due to the dominant quadratic nonlinearities [42]-[48]. Other works also reported nonlinear phenomena due to dominant quadratic nonlinearities in different compound structures [37], [39], and [47]-[49]. These studies demonstrated the importance of proper modeling with analytical and numerical techniques to understand their complex behavior. At the micro-scale, 


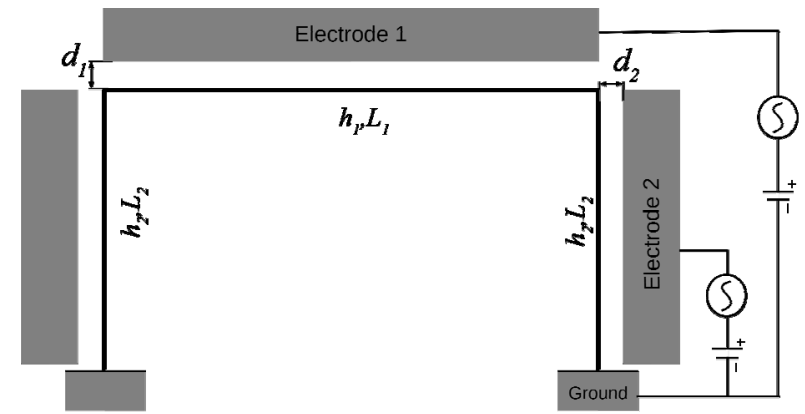

(a)

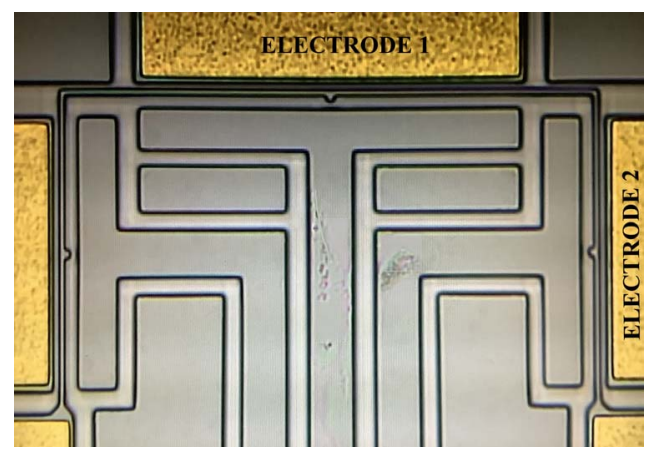

(b)

Fig. 1. (a) Schematic of the micro portal frame with the actuation electrodes; (b) Top view of the fabricated MEMS portal frame made of silicon.

U-shaped structures are very common for in-plane operations, such as electrothermal actuation [50], and logics [51]; as well as for out-of-plane operations, such as energy harvesting [52], and other linear and static applications.

Since at the micro-scale large deformations are commonly induced, it is essential to understand the dynamical behavior of compound structures while accounting properly for their nonlinear behavior. Specially, the portal frame has shown to have rich dynamical behaviors, such as the saturation phenomenon, the excitation of various higher order modes, and various ways to excite and tune resonance frequencies. It offers several design possibilities with a variety of electrode configurations for electrostatic actuation. Hence, it is important to model this system under such excitations to fully understand its behavior in order to effectively exploit it for different applications.

Hence, this work investigates the dynamical behavior of a micro portal frame designed to be excited at its first (sway, anti-symmetric) and second (symmetric) in-plane modes using DC and AC electrostatic forces.

\section{The Micro Portal Frame Design and Measurements Method}

The micro portal frame under study, shown in Figs. 1a, 1b, is fabricated by the SOIMUMPs process of MEMSCAP ${ }^{\mathrm{TM}}$ [53]. The microstructure is subjected to an electrostatic force through two electrodes, one on top of the supported beam (Electrode 1 (E1)) and the other on the column (Electrode 2 (E2). The device has Young Modulus of $136 \mathrm{GPa}$, the short beam elements (column) of length $L_{2}=133.1 \mu \mathrm{m}$, the long beam element (supported beam) of length $L_{1}=240 \mu \mathrm{m}$, and a depth of $25 \mu \mathrm{m}$. Due to imperfections from the fabrication process, the beam and columns widths are measured respectively as $h_{1}=1.73 \mu \mathrm{m}$, and $h_{2}=1.6 \mu \mathrm{m}$. The distance between the supported beam and columns from their respective electrodes are of $d_{1}=3.442 \mu \mathrm{m}$ and $d_{2}=3.51 \mu \mathrm{m}$, respectively.

It is important to mention that, due to the small gap between the resonator and the electrodes, a vacuum chamber is required to minimize squeeze-film damping [31] with the pressure of 50mTorr set throughout the experiments.

The resonance frequencies are measured using a laser Doppler vibrometer, Micro System Analyzer (MSA), from Polytec with white noise and very small AC voltage to get the Fast Fourier Spectrum FFT of the in-plane motion. To capture the forced vibration response, amplitude-frequency curves, stroboscopic video microscopy with a Bode plot of the MSA was used.

\section{Experimental Measurements and Finite ELEMENTS ANALYSIS}

The first and second natural frequencies were initially measured, as $79,580 \mathrm{~Hz}$ and $166,667 \mathrm{~Hz}$, respectively, as shown in Fig. 2. The first mode is actuated by Electrode 2 (E2) while Electrode 1 (E1) actuates the second mode. In addition, Figs. $2 \mathrm{a}$ and $2 \mathrm{~b}$ show the simulated modes of vibration of each frequency as obtained through a finite-element model using the software COMSOL.

Figs. $3 \mathrm{a}$ and $3 \mathrm{~b}$ show the frequency shift due to $V_{d c}$ for the first and second modes, respectively. They indicate that the natural frequencies of the structure decrease with the increase of the $V_{d c}$ due to the softening effect of the electrostatic force. When the first mode is actuated (see Fig. 3a), the second mode is slightly affected. However, when the second mode is actuated (see Fig. 3b), the first mode is barely affected.

Figure 3 also shows Finite Element Method (FEM) simulations using the software COMSOL Multiphysics. Good agreement is noted between the FEM and the experimental data. Both FEM results and experimental data show high tunability of the first mode when actuated by electrode E2 and of the second mode when actuated by electrode E1. Moreover, when actuating with E1, the second mode natural frequency crosses with the first one near the pull-in voltage (at $77.0 \mathrm{~V}$ ). The simulated maximum static displacements $\left(W_{\max }\right)$ of the portal frame just before pull-in of the first mode (Fig. 3a) and second mode (Fig. 3b) are $1.27 \mu \mathrm{m}$ and $1.35 \mu \mathrm{m}$, respectively; hence the ratio of $W_{\max }$ to gap electrode is around $37 \%$ in both cases.

The modes of vibration of compound structures are global modes representing the vibration of all connected single structures. In the portal frame, there are three beams coupled with each other. Each one of these beams has its own natural frequencies and corresponding modes of vibration. However, as the beams are mechanically coupled, they vibrate all at the same time with the same natural frequency. Here, the first global mode of vibration is dominated by the sway of the two columns, while the second mode is dominated by the bending of the clamped-clamped beam of the supported beam connecting the two columns. 


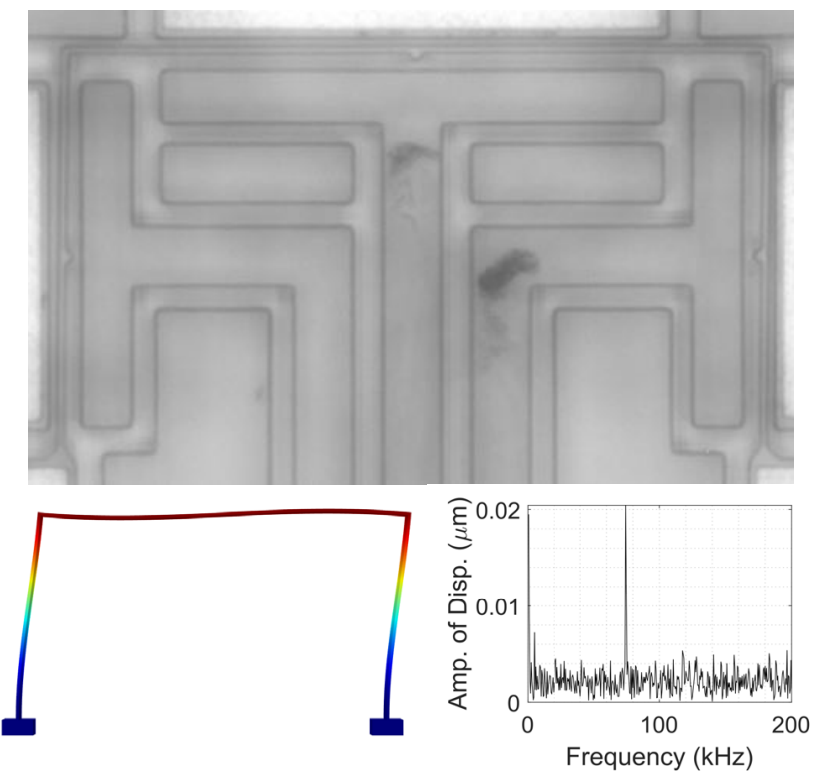

(a)
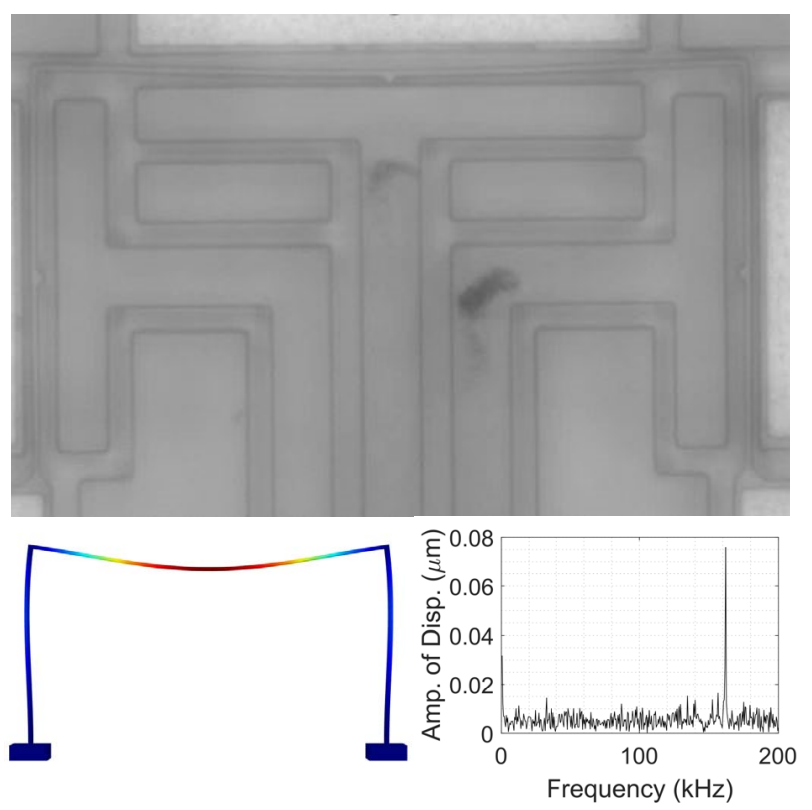

(b)

Fig. 2. Top views of the MEMS portal frame when vibrating at the (a) first and (b) second modes, with the measured Fast Fourier Transform (FFT), and finite element simulation of the modes.

Next, we use FEM simulations to study tuning the natural frequency of other higher-order modes of the micro-resonator under DC loads. The effect of various electrode configurations on these modes will be examined.

Table I shows the calculated natural frequencies at zero DC loads for the first five in-planes modes.

Figures $4 a, 4 b$ and $4 c$ show the FEM results of the frequency tuning for the third, fourth and fifth modes, respectively, when they are actuated by the electrode E2 or E1, as clarified in Fig. 1. Note that these electrodes affect the first and second modes as well. Hence, in the figure different ranges of frequency are shown for each used electrode as the first and second modes gets affected and eventually pulls-in. It is

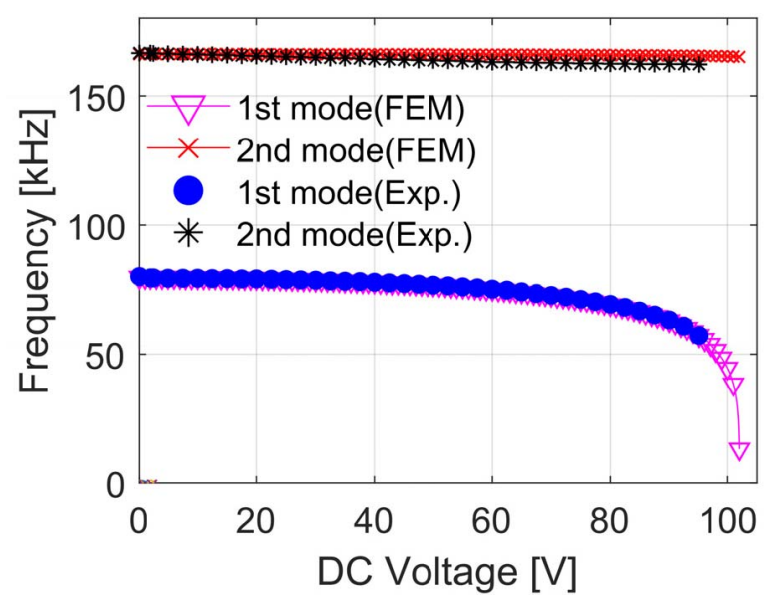

(a)

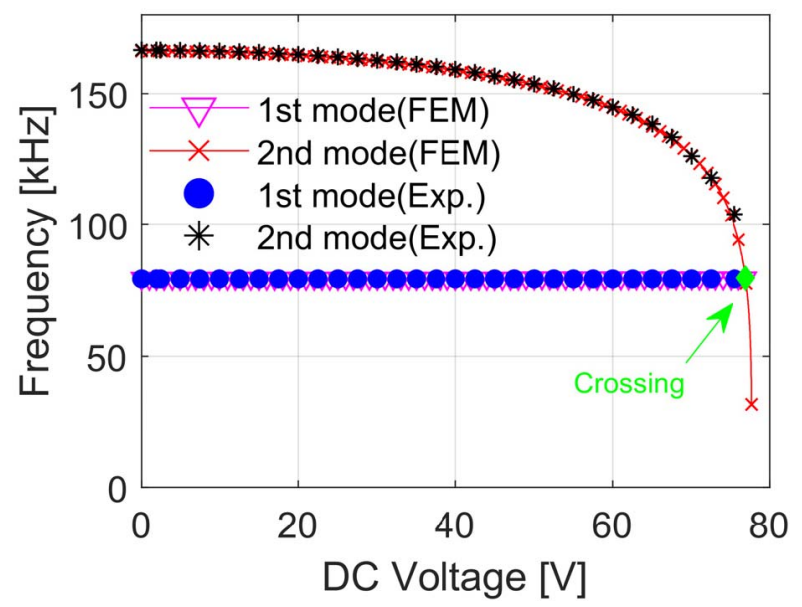

(b)

Fig. 3. Natural frequency shifts of the first two modes due to the effect of the electrostatic force, (a) when actuating the structure through Electrode 2 (E2) and (b) when actuating the structure with Electrode 1 (E1).

TABLE I

Natural Frequency of the First Five In-Planes Modes of Vibration of the Portal Frame Calculated Using FEM at Zero DC Voltage

\begin{tabular}{cc}
\hline \hline Modes of Vibration & Frequency $[\mathrm{kHz}]$ \\
\hline $1^{\text {st }}$ mode & 79.580 \\
$2^{\text {nd }}$ mode & 166.667 \\
$3^{\text {rd }}$ mode & 457.919 \\
$4^{\text {th }}$ mode & 613.811 \\
$5^{\text {th }}$ mode & 749.539 \\
\hline \hline
\end{tabular}

noticed that the higher-order modes have lower tunability than the first and second ones.

When E1 is activated, where the second mode pulls-in (as shown in Fig. 2b), there is slight decrease in the third and fifth modes (see Fig. 4a and 4c, respectively), while the fourth mode is barely affected (Fig. 4b). However, when E2 is activated, where the first mode pulls-in (as shown in Fig. 2a), the fourth mode is the most affected one, while the third almost does 


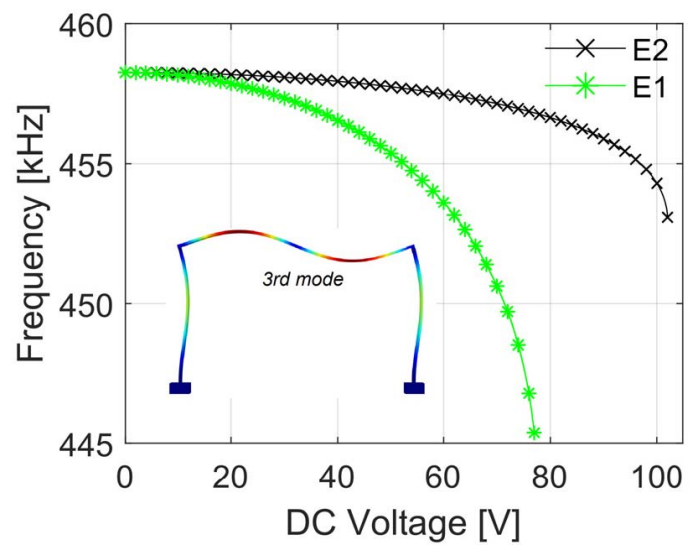

(a)

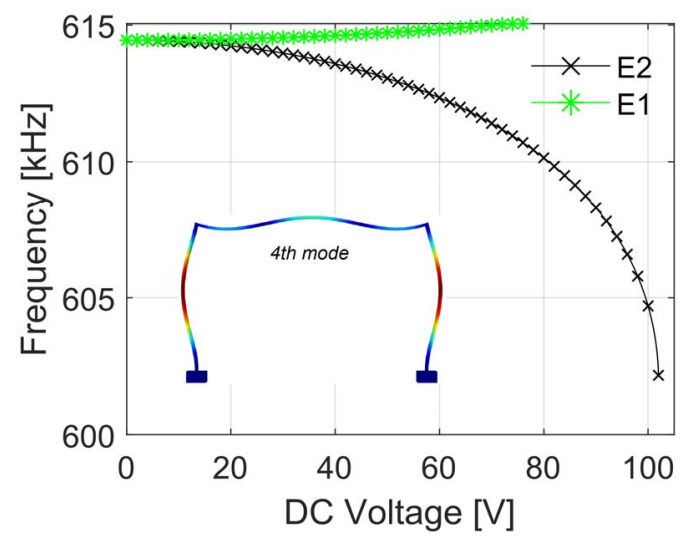

(b)

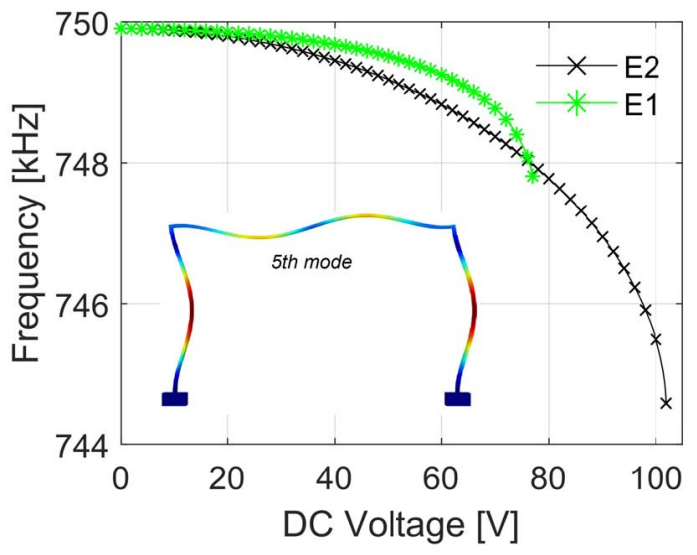

(c)

Fig. 4. Frequency shifting due to the effect of electrostatic force for (a) 3rd mode, (b) 4th mode, and (c) 5th mode when they are actuated by E1 or E2.

not decrease. Moreover, the fifth mode shifts more when E1 is activated.

Looking at the shapes of each mode, it is observed that the third mode depends on the motion of the long beam element $L_{1}$ of the frame. The fourth and fifth ones depend directly on the motion of the short beam ones $L_{2}$, clearing the fact that the actuation of the second mode affects most the third mode while the actuation of the first mode affects more the fourth and fifth ones.

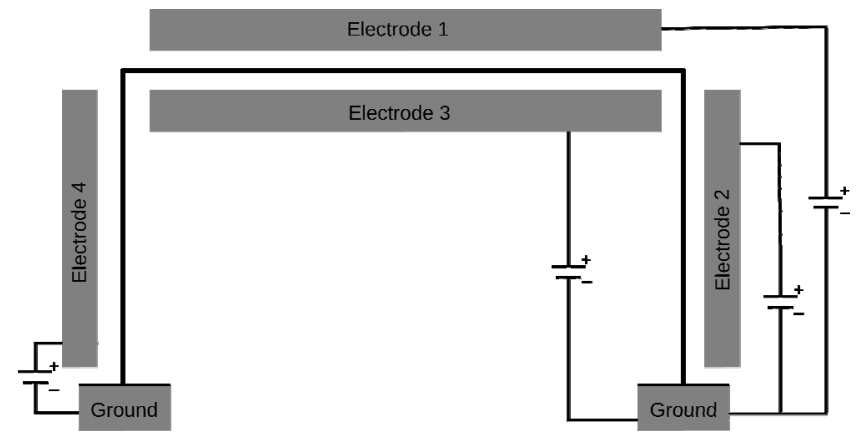

Fig. 5. Schematic illustrating various electrode configurations around the microstructure.

\section{EFFECT OF Other ElECTROde CONFIGURATIONS ON HIGHER-ORDER MODES}

The effect of different electrode configurations is investigated next on the natural frequency of higher-order modes of vibration. Figure 5 shows a schematic for the various studied electrode configurations. In addition to the initial design (Fig. 1b), two more electrodes are considered in the opposite side of E1 and E2, named E3 and E4.

Figure 6 shows the frequency shifts of the first five in-plane modes for all the different configurations of electrodes. The numbers in the legends indicates the used configurations.

The first and second natural frequencies are the most affected by the electrostatic force. For all the configurations, either the first or the second mode pulls-in first.

As noted from Fig. $3 a$ and 3b, an electrode configuration can have significant impact on one mode while it barely affects the other. Then, looking at the case of actuation of E1 and E2 at the same time (case 1-2 in Fig. 6), both first and second modes have their natural frequencies shifted. The figure also indicates that all the higher-order modes are affected by the electrostatic force. The most affected one is the third mode, as the long element $L_{1}$ of the portal frame is less stiff.

Furthermore, to shift all the natural frequencies and increase the tunability of the higher-order modes, the case of actuating the structure with all the four electrodes E1, E2, E3 and E4 (case 1-2-3-4 in Fig. 6) is the best among them. The $2^{\text {nd }}$ mode is the first one to pull-in and the 3rd, 4th and 5th modes are all affected, which is obvious as the whole structure is actuated at the same time. The reason that the second mode pulls-in before the other modes is that this mode depends on the long element, which is less stiff than the other ones.

When the structure is actuated by E1 and E3 (case 1-3), the frequency tunability of higher-order modes is enhanced compared to case 1 , as shown in Figs. $6 c, 6 d$ and $6 e$. The natural frequencies of higher-order modes decrease even more with the increase of DC voltage. The pull-in voltage of the second mode increases around 25\%, as shown in Fig. 6b.

Similarly, the tunability of case $2-4$ compared to case 2 is enhanced for higher-order modes, as shown in Figs. 6c, 6d and $6 \mathrm{e}$. In addition, the pull-in voltage of the first mode increases around $25 \%$, as shown in Fig. 6a. This is because the structure is actuated by two electrodes on the same element. 


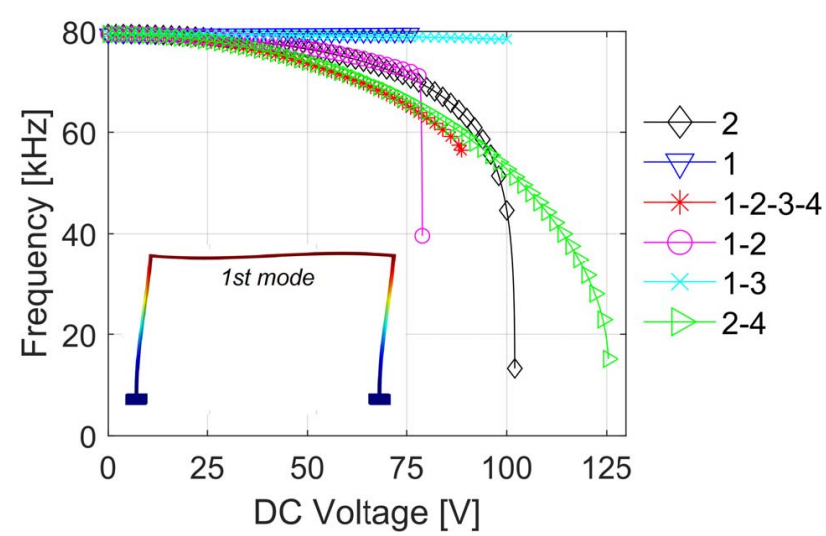

(a)

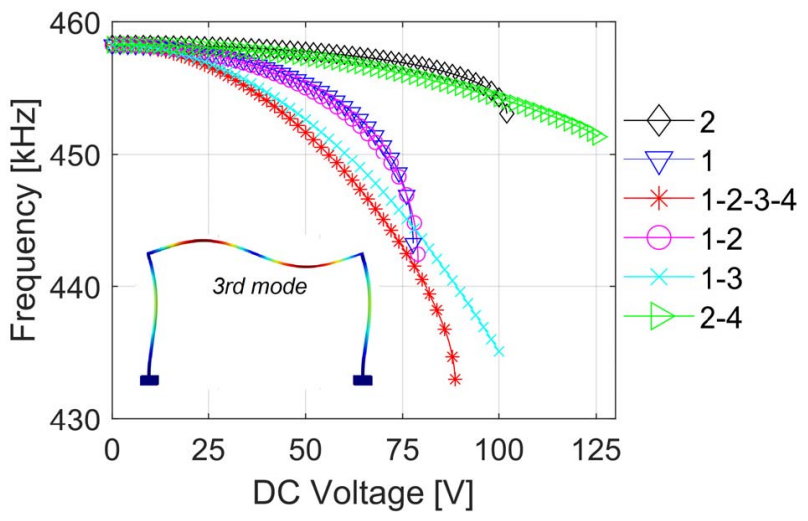

(c)

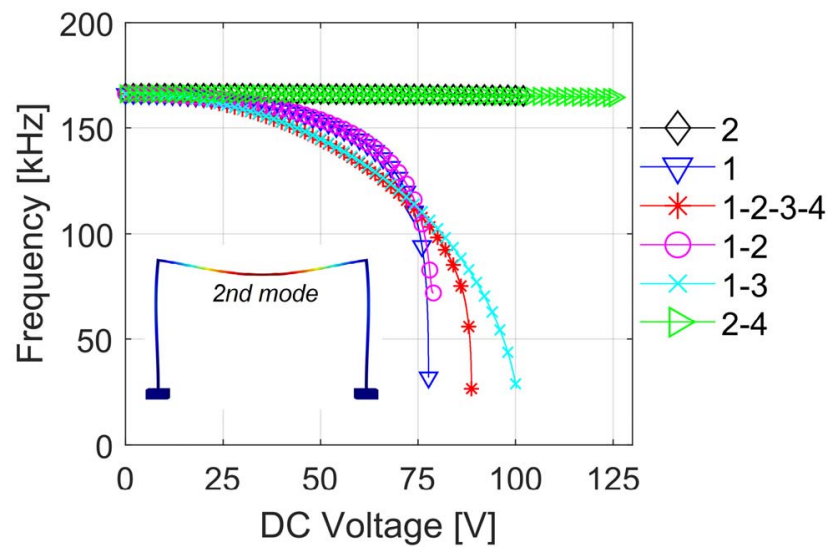

(b)

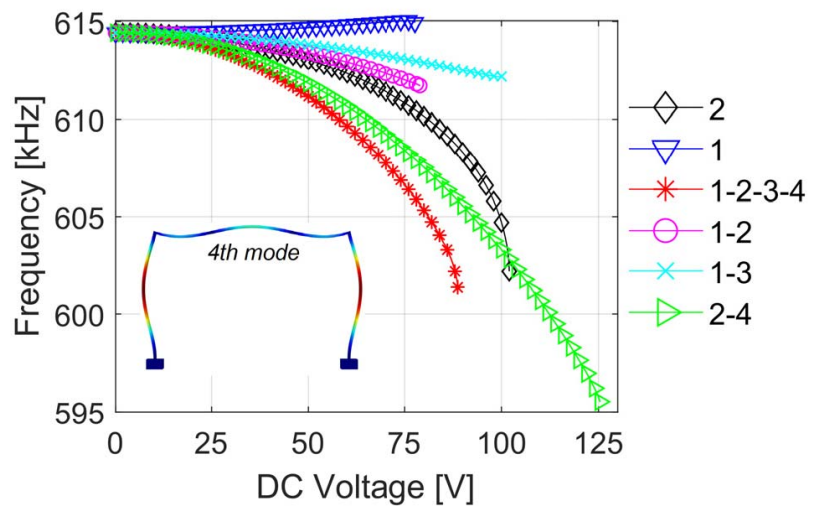

(d)

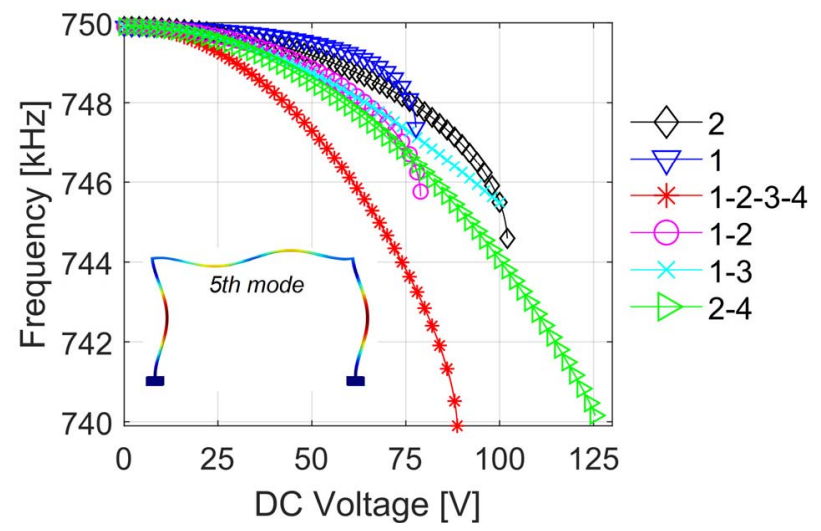

(e)

Fig. 6. Frequency shifts of (a) 1st mode, (b) 2nd mode, (c) 3rd mode, (d) 4th mode, and (e) 5 th mode, for different electrode configurations. The numbers on the legends represent the number of the used electrodes as indicated in Fig. 5.

It is noticed from Figs. 3 and 6 that the 2nd mode natural frequency can decrease until it reaches a value close to the 1st mode natural frequency. Fig. 7 shows what happens when the 2nd mode is close to the first mode for the cases where the 2nd mode is the most affected. All the curves starting near $120 \mathrm{kHz}$ are for the second mode while the curves starting around $75 \mathrm{kHz}$ of frequency are for the first mode.

When the system is actuated by E1 only (case 1), E1 to E4 (case 1-2-3-4), and E1 and E3 (case 1-3), the second mode crosses the first mode. However, when the system is actuated by E1 and E2 (case 1-2), it is possible to see that the modes do not cross. The second mode deviates (veers away) when the first one sudden drops to pull-in. It is clear from Fig. 7 that this indicates a veering phenomenon [54]. This is due to the effect of electrostatic force in breaking the symmetry of the structure. The electrodes E1 and E2 actuating on the long element $L_{1}$ and one of the short elements $L_{2}$, respectively, soften the elements, making them less stiff than the other short 


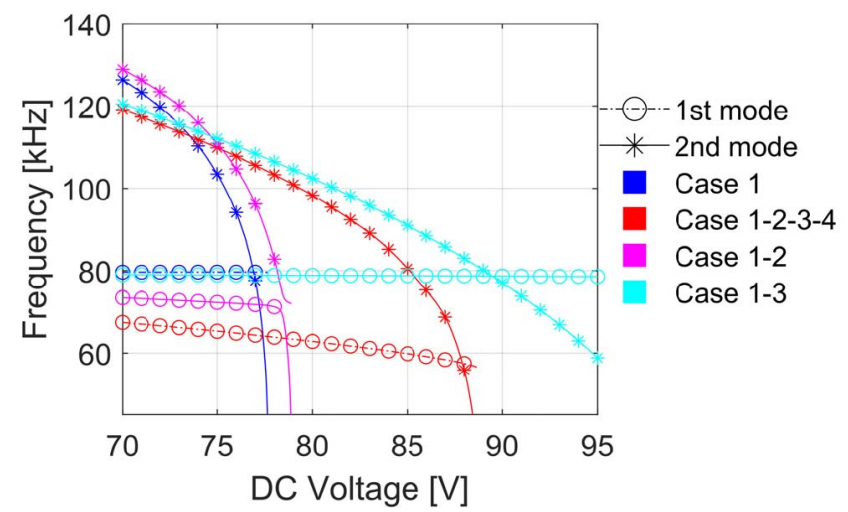

Fig. 7. Frequency shifting of the first mode and second mode for the cases where the second mode is tuned more than the first mode around the crossings. The dotted line with circles indicates the first modes, and the solid line with stars indicates the second modes. The colors of the lines indicate the cases of the electrodes' configuration.

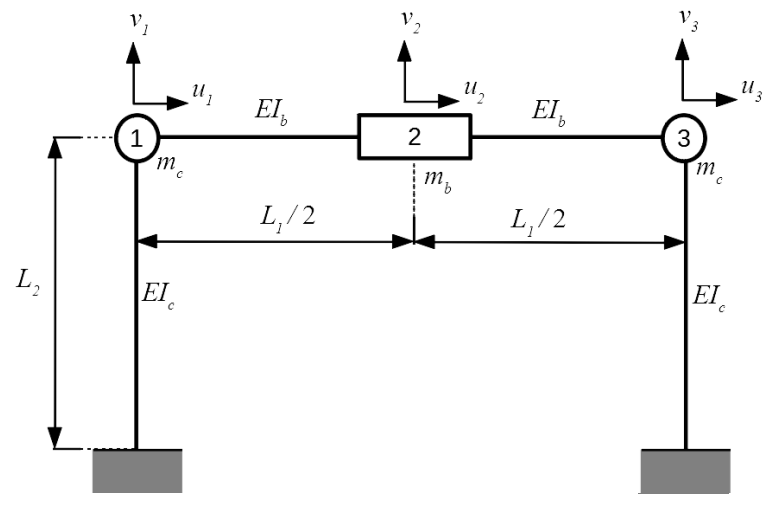

Fig. 8. Schematic of the micro portal frame model.

element. With that, the symmetry between both sides of the portal frame is broken.

\section{NONLINEAR DYNAMIC ANALYSis}

\section{A. The Model}

Next, a mathematical model is developed to simulate the dynamic response of the system. For this, the micro portal frame of Fig. 1a is represented by Fig. 8. The supported middle beam is represented by the lumped mass $m_{b}$ and a moment of inertia $I_{b}$. Each vertical column is represented by a lumped masses $m_{c}$ with a moment of inertia $I_{c}$. Both columns and beam have a depth $b$. All the other parameters are presented in Section II. In the reduced-order mathematical model, inextensible links are considered.

The linear stiffness of the columns and the beam can be evaluated by a finite-element Rayleigh-Ritz procedure using cubic trial functions [45]. The shortening due to bending of the columns and beam introduces quadratic geometric nonlinearity of the structure. The shape functions related to the columns and beam can be approximated as

$$
\begin{array}{ll}
\phi_{u}=\frac{6}{L_{2}^{3}}\left(\frac{L_{2} x^{2}}{2}-\frac{x^{3}}{6}\right), & 0 \leq x \leq L_{2} \\
\phi_{v}=\frac{12}{L_{1}^{3}}\left(\frac{x^{3}}{3}-\frac{L_{1}^{2} x}{4}\right), & 0 \leq x \leq L_{1}
\end{array}
$$

where $\phi_{u}$ describes an interpolated shape function of the first mode of a cantilever beam along the column length $L_{2}$ and $\phi_{v}$ describes an interpolated shape function of the first mode of a clamped-clamped beam along half of the supported beam length $L_{1}$.

The nodal displacements, shown in Fig. 8, are given by

$$
\begin{aligned}
& u_{2}=q_{1} \quad u_{1}=u_{2}+\frac{B}{2} v_{2}^{2} \quad u_{3}=u_{2}-\frac{B}{2} v_{2}^{2} \\
& v_{2}=q_{2} \quad v_{1}=-\frac{C}{2} u_{2}^{2} \quad v_{3}=-\frac{C}{2} u_{2}^{2}
\end{aligned}
$$

where the constants $B$ and $C$ are as below

$$
C=\int_{0}^{L_{2}}\left(\phi_{u}^{\prime}\right)^{2} d x \quad B=\int_{0}^{L_{1}}\left(\phi_{v}^{\prime}\right)^{2} d x
$$

We calculate the linear stiffness of the beam $k_{b}$ and columns $k_{c}$ through the Rayleigh's method as

$$
k_{c}=E I_{c} \int_{0}^{L_{2}}\left(\phi_{u}^{\prime \prime}\right)^{2} d x \quad k_{b}=E I_{b} \int_{0}^{L_{1}}\left(\phi_{v}^{\prime \prime}\right)^{2} d x
$$

This yields $B=69 / 5 L_{1}, C=24 / 5 L_{2}, k_{b}=192 E I_{b} / L_{1}^{3}$, and $k_{c}=12 E I_{c} / L_{2}^{3}$. Note that the generalized coordinates of the structure are considered the motion of the mass at the mid-span of the supported beam; $u_{2}=q_{1}$, and $v_{2}=q_{2}$, as in Eq. (2), and illustrated in Fig. 8.

The total kinetic energy of the structure $T$ is calculated by accounting for the motion of all the three masses related to the nodal displacement as

$$
T=\frac{1}{2} m_{b}\left(\dot{u}_{2}^{2}+\dot{v}_{2}^{2}\right)+\frac{1}{2} m_{c}\left(\dot{u}_{1}^{2}+\dot{u}_{3}^{2}+\dot{v}_{1}^{2}+\dot{v}_{3}^{2}\right)
$$

where the masses are calculated by

$$
m_{c}=\rho b h_{2} \int_{0}^{L_{2}} \phi_{u}^{2} d x ; \quad m_{b}=\rho b h_{1} \int_{0}^{L_{1}} \phi_{v}^{2} d x
$$

where $\rho$ is the mass density of the material. Hence, deriving Eq. (2) in time and substituting into Eq. (4) yields

$T=\frac{1}{2} m_{b}\left(\dot{q}_{1}^{2}+\dot{q}_{2}^{2}\right)+m_{c} \dot{q}_{1}^{2}+C^{2} m_{c} q_{1}^{2} \dot{q}_{1}^{2}+B^{2} m_{c} q_{2}^{2} \dot{q}_{2}^{2}$

Note that from Eq. (6), inertia nonlinearities are obtained. The potential energy of the structure $U$ is given by

$$
U=\frac{1}{2} k_{c}\left(u_{1}^{2}+u_{3}^{2}\right)+\frac{1}{2} k_{b}\left(v_{2}-\frac{v_{1}+v_{3}}{2}\right)^{2}
$$

Substituting the nodal displacements of Eq. (2) into Eq. (7) yields

$$
U=k_{c} q_{2}^{2}+\frac{1}{2} C k_{b} q_{1} q_{2}^{2}+\frac{1}{2} k_{b} q_{1}^{2}+\frac{1}{4} C^{2} k_{b} q_{2}^{4}+\frac{1}{8} B^{2} k_{c} q_{1}^{4}
$$


The potential energy shows quadratic, cubic, quartic, and coupling terms. These nonlinear terms may drive the system into nonlinear behaviors.

The dissipative energy $D$ of the supported beam and columns are defined as

$$
D=\frac{1}{2} 2 c_{c} \dot{q}_{1}^{2}+\frac{1}{2} c_{b} \dot{q}_{2}^{2}
$$

where $c_{c}$ and $c_{b}$ are the damping coefficients of the columns and supported beam, respectively.

The structure is actuated by DC and AC electrostatic loads through Electrode 1 and Electrode 2. Hence, the electrostatic force for each electrode is given by

$$
\begin{aligned}
& F_{e_{q 1}}=\frac{\varepsilon A_{1}}{2\left(d_{2}-u_{1}\right)^{2}}\left(V_{d c 1}+V_{a c 1} \cos \omega_{1} t\right)^{2} \\
& F_{e_{q 2}}=\frac{\varepsilon A_{2}}{2\left(d_{1}-v_{2}\right)^{2}}\left(V_{d c 2}+V_{a c 2} \cos \omega_{2} t\right)^{2}
\end{aligned}
$$

where the indexes 1 and 2 are related to the coordinates $q_{1}$ and $q_{2}$. Note that the quadratic term of $u_{1}$ is neglected in Eq. (10) as its influence is negligible for the results.

Next, we apply the Euler-Lagrange equations

$$
\begin{aligned}
L(q, \dot{q}, t) & =T-U \\
\frac{d}{d t}\left(\frac{\partial L}{\partial \dot{q}_{i}}\right)-\frac{\partial L}{\partial q_{i}}+\frac{\partial D}{\partial \dot{q}_{i}} & =Q_{\text {ext }} \quad i=1,2
\end{aligned}
$$

where $Q_{\text {ext }}$ is the generalized non-conservative forces.

Hence, the equations of motion of the portal frame are obtained as

$$
\begin{aligned}
\left(2 m_{c}\right. & \left.+m_{b}\right) \ddot{q}_{1}+c_{c} \dot{q}_{1}+2 k_{c} q_{1}+2 C^{2} m_{c}\left(q_{1}^{2} \ddot{q}_{1}+q_{1} \dot{q}_{1}^{2}\right) \\
& +C k_{b} q_{1} q_{2}+\frac{1}{2} C^{2} k_{b} q_{1}^{3}=\frac{\varepsilon A_{1}\left(V_{d c 1}+V_{a c 1} \cos \omega_{1} t\right)^{2}}{2\left(d_{2}-q_{1}\right)^{2}} \\
m_{b} \ddot{q}_{2} & +c_{b} \dot{q}_{2}+k_{b} q_{2}+2 B^{2} m_{c}\left(q_{2}^{2} \ddot{q}_{2}+q_{1} \dot{q}_{2}^{2}\right) \\
& +\frac{1}{2} C k_{b} q_{1}^{2}+B^{2} k_{c} q_{2}^{3}=\frac{\varepsilon A_{2}\left(V_{d c 2}+V_{a c 2} \cos \omega_{2} t\right)^{2}}{2\left(d_{1}-q_{2}\right)^{2}}
\end{aligned}
$$

where the first and second equations represent the horizontal and vertical displacements, respectively, of the mass at the mid-span of the supported beam.

A dimensionless procedure is carried out with the new nondimensional variables below

$$
x=\frac{q_{1}}{d_{2}} ; \quad y=\frac{q_{2}}{d_{1}} ; \quad \omega_{n 1}=\sqrt{\frac{2 k_{c}}{2 m+M}} ; \quad \tau=\omega_{n 1} t
$$

yielding the new non-dimensional equations of motion:

$$
\begin{aligned}
\ddot{x}+ & \mu_{1} \dot{x}+x+k_{31} x^{3}+\alpha_{11} x y+\alpha_{21}\left(x \dot{x}^{2}+x^{2} \ddot{x}\right) \\
& =E_{1} \frac{\left(V_{d c 1}+V_{a c 1} \cos \Omega_{1} \tau\right)^{2}}{(1-x)^{2}} \\
\ddot{y}+ & \mu_{2} \dot{y}+\omega_{n 2}^{2} y+k_{32} y^{3}+\alpha_{12} x^{2}+\alpha_{22}\left(y \dot{y}^{2}+y^{2} \ddot{y}\right) \\
& =E_{2} \frac{\left(V_{d c 2}+V_{a c 2} \cos \Omega_{2} \tau\right)^{2}}{(1-y)^{2}}
\end{aligned}
$$

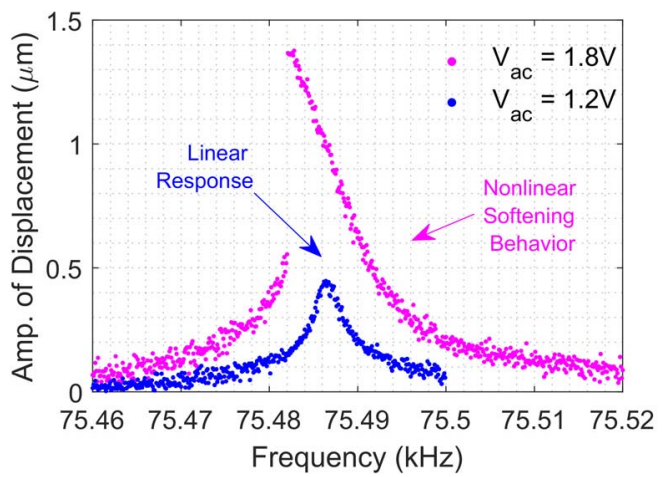

(a)

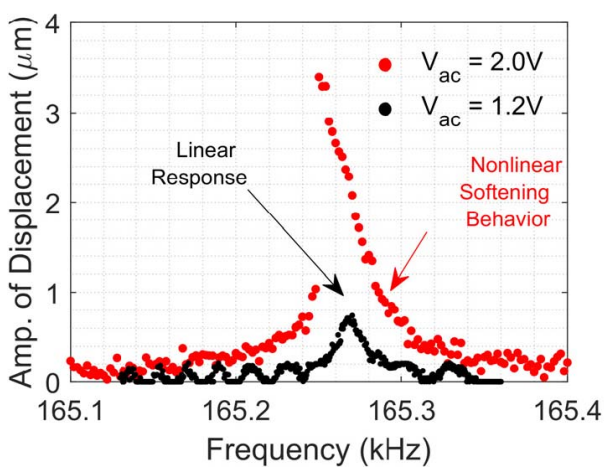

(b)

Fig. 9. Experimental frequency-response curves of the (a) $1^{\text {st }}$ mode and (b) $2^{\text {nd }}$ mode, respectively.

where the dimensionless coefficients are:

$$
\begin{aligned}
k_{31} & =\frac{C^{2} d_{2}^{2} k_{b}}{2\left(m_{b}+2 m_{c}\right) \omega_{n 1}^{2}} ; \alpha_{11}=\frac{C d_{1} k_{b}}{\left(m_{b}+2 m_{c}\right) \omega_{n 1}^{2}} ; \\
\alpha_{21} & =\frac{2 C^{2} d_{2}^{2} m_{c}}{m_{b}+2 m_{c}} ; \quad \mu_{1}=\frac{c_{c}}{\left(m_{b}+2 m_{c}\right) \omega_{n 1}} ; \Omega_{1}=\frac{\omega_{1}}{\omega_{n 1}} ; \\
E_{2} & =\frac{\varepsilon A_{2}}{2 d_{1}^{3} m_{b} \omega_{n 1}^{2}} ; \quad E_{1}=\frac{\varepsilon A_{1}}{2 d_{2}^{3}\left(m_{b}+2 m_{c}\right) \omega_{n 1}^{2}} ; \\
k_{32} & =\frac{B^{2} d_{1}^{2} k_{c}}{m_{b} \omega_{n 1}^{2}} ; \omega_{n 2}=\sqrt{\frac{k_{b}}{m_{b} \omega_{n 1}^{2}}} ; \alpha_{12}=\frac{C d_{2}^{2} k_{b}}{2 d_{1} m_{b} \omega_{n 1}^{2}} ; \\
\alpha_{22} & =\frac{2 B^{2} d_{2}^{2} m_{c}}{m_{b}} ; \quad \mu_{2}=\frac{c_{b}}{m_{b} \omega_{n 1}} ; \quad \Omega_{2}=\frac{\omega_{2}}{\omega_{n 1}} ;
\end{aligned}
$$

\section{B. Experimental Results}

The first and second modes are excited by an AC voltage excitation with no DC voltage. This is to suppress the softening effect of the DC voltage due to the quadratic nonlinearity [31]; and hence highlight the effect of other nonlinearities from the geometry of the structure itself.

The experimental frequency response curves of the first anti-symmetric ( $1^{\text {st }}$ mode) and first symmetric ( $2^{\text {nd }}$ mode) modes are depicted in Figs. 9a and 9b, respectively. Linear responses of each mode are noted when applying a very small AC voltage excitation $\left(V_{a c}=1.2 \mathrm{~V}\right)$. However, with a small increase in the $\mathrm{AC}$ voltage $\left(V_{a c}=1.8 \mathrm{~V}\left(1^{\text {st }}\right.\right.$ mode $)$ and $2.0 \mathrm{~V}$ $\left(2^{\text {nd }}\right.$ mode $)$ ), one can see nonlinear softening behavior. Since 


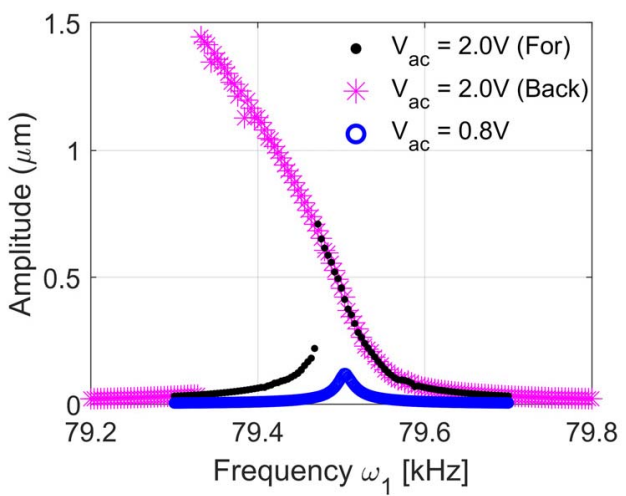

(a)

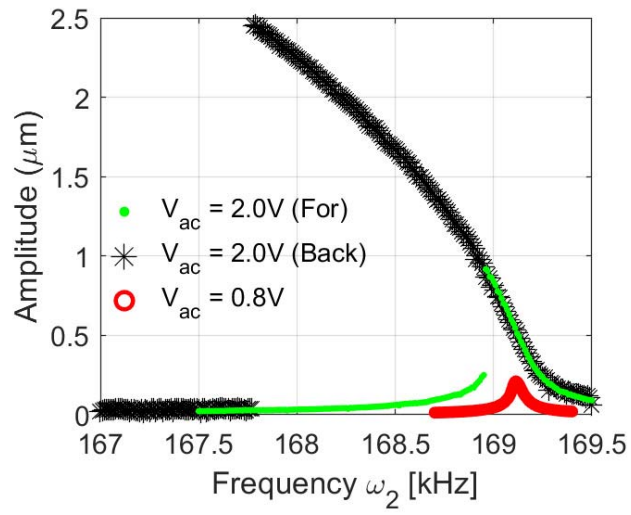

(b)

Fig. 10. Simulated frequency-response curves of the (a) $1^{\text {st }}$ mode and (b) $2^{\text {nd }}$ mode.

there is no $V_{d c}$ and very small $V_{a c}$ excitation, it is clear that the softening behavior is purely due to geometric nonlinearities from the frame, providing unique and interesting dynamics of this microstructure.

\section{Simulation Results}

Integrating Eqs. (15) in time, the steady-state response of the system is obtained assuming $c_{c}=c_{b}=20.1 \mathrm{pNs} / \mathrm{m}$ and $V_{d c 1}=V_{d c 2}=0 \mathrm{~V}$. The simulated frequency response curves of the first and second modes are shown in Figs. 10a and 10b, respectively. As in the experimental curves, linear responses are noted when applying a small $\mathrm{AC}$ voltage excitation $\left(V_{a c 1}=\right.$ $\left.V_{a c 2}=0.8 \mathrm{~V}\right)$. With a small increase in the AC voltage excitation, $V_{a c 1}=2.0 \mathrm{~V}\left(1^{\text {st }}\right.$ mode $)$ and $V_{a c 2}=2.0 \mathrm{~V}\left(2^{\text {nd }}\right.$ mode), forward and backward integrations are carried out. For both modes there is nonlinear softening behavior.

The investigation of the geometry of the portal frame is carried out by observing the frequency-responses of both $1^{\text {st }}$ and $2^{\text {nd }}$ modes for different lengths of the supported beam $L_{1}$ that are considered as $L_{1}=180 \mu \mathrm{m}, 220 \mu \mathrm{m}$, and $290 \mu \mathrm{m}$. The smaller is $L_{1}$, the higher is the natural frequencies of the modes.

Fig. 11 shows various frequency-response curves accounting for the different lengths of the supported beam, from $L_{1}=$ $180 \mu \mathrm{m}$ (Fig. 11a) to $L_{1}=290 \mu \mathrm{m}$ (Fig. 11c), respectively, excited by a $V_{a c 1}=V_{a c 2}=2.0 \mathrm{~V}$, except the last one (Fig. 11c, with $V_{a c 1}=V_{a c 2}=1.3 \mathrm{~V}$ ). The figures in the left side
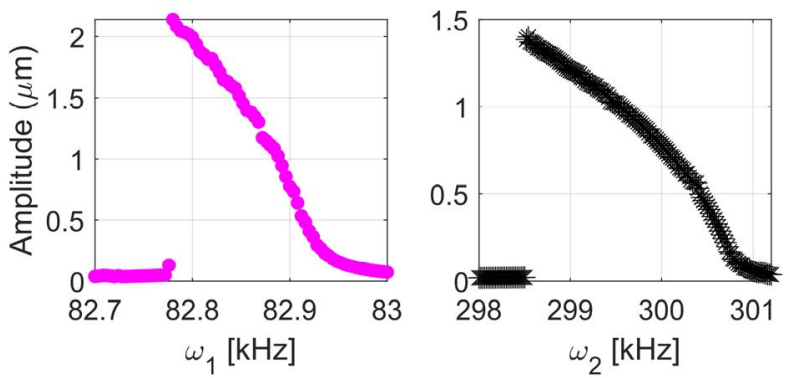

(a)

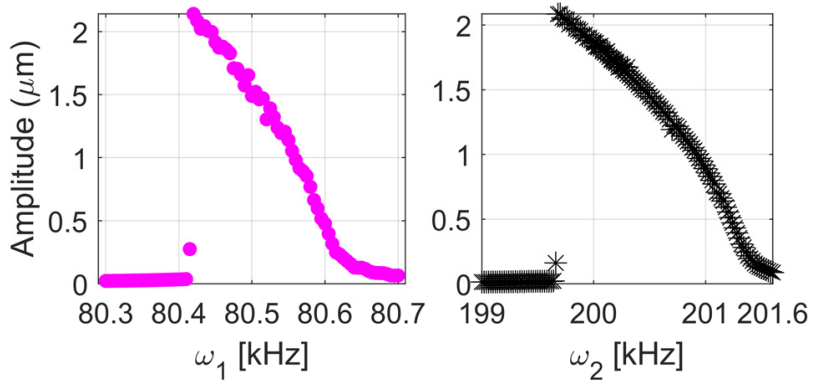

(b)
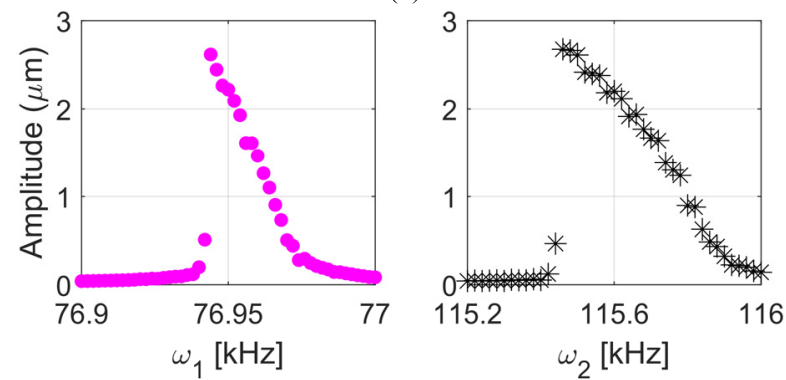

(c)

Fig. 11. Simulated frequency-response curves of the $1^{\text {st }}$ (left figures) and $2^{\text {nd }}$ (right figures) modes, respectively, for the supported beam length $L_{1}$ of (a) $180 \mu \mathrm{m}$ for $V_{a c 1}=V_{a c 2}=2.0 \mathrm{~V}$, (b) $220 \mu \mathrm{m}$ for $V_{a c 1}=V_{a c 2}=2.0 \mathrm{~V}$, and (c) $290 \mu \mathrm{m}$ for $V_{a c 1}=V_{a c 2}=1.3 \mathrm{~V}$. All simulations show backward sweep.

represent the $1^{\text {st }}$ mode frequency-responses while the right side figures are for the $2^{\text {nd }}$ mode ones.

With the increase of $L_{1}$, it is observed the increase of the final response of the softening behavior of the $2^{\text {nd }}$ mode, while the $1^{\text {st }}$ mode seems to decrease (see the progression of the curves for Fig. 11a, 11b, 10a and 10b).

However, when $L_{1}=290 \mu \mathrm{m}$, the $1^{\text {st }}$ mode response increases again. Note that Fig. 11c is simulated for $V_{a c 1}=$ $V_{a c 2}=1.3 \mathrm{~V}$. As expected, the longer is the beam the higher is the amplitude of vibration. Then, dynamic pull-in is observed at $V_{a c 1}=V_{a c 2}=2.0 \mathrm{~V}$.

Since the modeling of the portal frame accounts for quadratic and cubic geometric nonlinearities, the quadratic ones seem dominant as observed in the various different geometries of the structure.

\section{CONCLUSION}

An experimental and FEM study of the tunability of an inplane micro-electromechanical portal frame (U-shape) structure under DC electrostatic excitation has been presented. The results showed high tunability for the first symmetric and first anti-symmetric (sway) modes of vibration. 
FEM results showed the possibility to tune higher-order modes. Even though they are of low tunability, the third mode can be better tuned when the supported beam $L_{1}$ is excited, while the fourth and fifth modes can be effectively tuned by exciting the columns $L_{2}$. One way to have a slightly high tunability of the higher-order modes is exciting the system with different configurations of the electrodes on the structure. Crossings and veering between the first and second modes can be observed during various tunability scenarios. However, all the crossing and the veering phenomena were very close to the pull-in voltage.

Besides that, it is also possible to clarify that the direct actuation on the supported beam $L_{1}$ tunes the third mode, while the fourth and fifth modes are tuned only when the columns $L_{2}$ are actuated.

Moreover, the structure was experimentally excited sweeping the frequency near the resonance only with electrostatic AC voltage. The frequency response curves showed linear response for a very small voltage $(1.0 \mathrm{~V})$. However, increasing the voltage to $V_{a c}=2.0 \mathrm{~V}$, the system showed nonlinear softening behavior, which clearly comes from the geometric nonlinearities of the structure, as there was no DC load excitation.

A mathematical model for the first and second modes was also developed and showed the dominance of the quadratic nonlinearity of the portal frame. This highlights the fact that the portal frame has an interesting dynamic due to the structure itself.

The results of this paper shed light on the possibility of studying and utilizing other compound structures with multiple excitation inputs and multiple outputs, which can be used for variety of applications, such as in logic, computation [55], sensing, and actuations. These results open possibilities for applications, such as in logic gates and switches due to the tuning between those two modes, including the different ways that they can be actuated and sensed. Also, the system showed rich nonlinear dynamics with high potential for applications in energy harvesting due to the low energy necessary to access high amplitudes of vibration.

\section{REFERENCES}

[1] A. Z. Hajjaj, N. Jaber, N. Alcheikh, and M. I. Younis, "A sensitive resonant gas sensor based on multimode excitation of a buckled beam," in Proc. 24th Design Manuf. Life Cycle Conf., 13th Int. Conf. MicroNanosyst., Aug. 2019, pp. 769-772.

[2] J. Kehrbusch, P. Bozek, B. Radzio, E. A. Ilin, and E. Oesterschulze, "Columnar shaped microresonators for mass detection and gas analysis," Microelectron. Eng., vol. 87, nos. 5-8, pp. 817-820, May 2010.

[3] A. Bouchaala, A. H. Nayfeh, N. Jaber, and M. I. Younis, "Mass and position determination in MEMS mass sensors: A theoretical and an experimental investigation," J. Micromech. Microeng., vol. 26, no. 10, Oct. 2016, Art. no. 105009.

[4] D. C. Abeysinghe, S. Dasgupta, J. T. Boyd, and H. E. Jackson, "A novel MEMS pressure sensor fabricated on an optical fiber," IEEE Photon. Technol. Lett., vol. 13, no. 9, pp. 993-995, Sep. 2001.

[5] E. Timurdogan, B. E. Alaca, I. H. Kavakli, and H. Urey, "MEMS biosensor for detection of hepatitis a and c viruses in serum," Biosensors Bioelectron., vol. 28, no. 1, pp. 189-194, Oct. 2011.

[6] S. Yang and Q. Xu, "A review on actuation and sensing techniques for MEMS-based microgrippers," J. Micro-Bio Robot., vol. 13, nos. 1-4, pp. 1-14, Oct. 2017.

[7] R. R. A. Syms, H. Zou, J. Yao, D. Uttamchandani, and J. Stagg, "Scalable electrothermal MEMS actuator for optical fibre alignment," J. Micromech. Microeng., vol. 14, no. 12, pp. 1633-1639, Dec. 2004.
[8] K. A. Cook-Chennault, N. Thambi, and A. M. Sastry, "Powering MEMS portable devices-A review of non-regenerative and regenerative power supply systems with special emphasis on piezoelectric energy harvesting systems," Smart Mater. Struct., vol. 17, no. 4, Aug. 2008, Art. no. 043001.

[9] J.-Q. Liu et al., "A MEMS-based piezoelectric power generator array for vibration energy harvesting," Microelectron. J., vol. 39, no. 5, pp. 802-806, May 2008.

[10] G. M. Rebeiz and J. B. Muldavin, "RF MEMS switches and switch circuits," IEEE Microw. Mag., vol. 2, no. 4, pp. 59-71, Dec. 2001.

[11] S. Molaei and B. A. Ganji, "Design and simulation of a novel RF MEMS shunt capacitive switch with low actuation voltage and high isolation," Microsyst. Technol., vol. 23, no. 6, pp. 1907-1912, Jun. 2017.

[12] S. A. Tella, N. Alcheikh, and M. I. Younis, "A single MEMS resonator for reconfigurable multifunctional logic gates," J. Micromech. Microeng., vol. 28, no. 9, Sep. 2018, Art. no. 095002.

[13] H. Samaali, Y. Perrin, A. Galisultanov, H. Fanet, G. Pillonnet, and P. Basset, "MEMS four-terminal variable capacitor for low power capacitive adiabatic logic with high logic state differentiation," Nano Energy, vol. 55, pp. 277-287, Jan. 2019.

[14] A. Pothier et al., "Low-loss 2-bit tunable bandpass filters using MEMS DC contact switches," IEEE Trans. Microw. Theory Techn., vol. 53, no. 1, pp. 354-360, Jan. 2005.

[15] R. Xu, S. Zhou, and W. J. Li, "MEMS accelerometer based nonspecificuser hand gesture recognition," IEEE Sensors J., vol. 12, no. 5, pp. 1166-1173, May 2012.

[16] M. I. Younis and A. H. Nayfeh, "A study of the nonlinear response of a resonant microbeam to an electric actuation," Nonlinear Dyn., vol. 31, no. 1, pp. 91-117, Jan. 2003.

[17] A. M. Tusset, J. M. Balthazar, D. G. Bassinello, B. R. Pontes, and J. L. P. Felix, "Statements on chaos control designs, including a fractional order dynamical system, applied to a 'MEMS' comb-drive actuator," Nonlinear Dyn., vol. 69, no. 4, pp. 1837-1857, Sep. 2012.

[18] F. K. Alfosail, A. Z. Hajjaj, and M. I. Younis, "Theoretical and experimental investigation of two-to-one internal resonance in MEMS arch resonators," J. Comput. Nonlinear Dyn., vol. 14, no. 1, pp. 1-43, Jan. 2019.

[19] M. H. Ghayesh, H. Farokhi, and M. Amabili, "Nonlinear behaviour of electrically actuated MEMS resonators," Int. J. Eng. Sci., vol. 71, pp. 137-155, Oct. 2013.

[20] H. M. Ouakad and M. I. Younis, "The dynamic behavior of MEMS arch resonators actuated electrically," Int. J. Non-Linear Mech., vol. 45, no. 7, pp. 704-713, Sep. 2010.

[21] W. Zhang, R. Baskaran, and K. L. Turner, "Effect of cubic nonlinearity on auto-parametrically amplified resonant MEMS mass sensor," Sens. Actuators A, Phys., vol. 102, nos. 1-2, pp. 139-150, Dec. 2002.

[22] A. Yao and T. Hikihara, "Counter operation in nonlinear micro-electromechanical resonators," Phys. Lett. A, vol. 377, no. 38, pp. 2551-2555, Nov. 2013.

[23] S. Krylov, B. R. Ilic, D. Schreiber, S. Seretensky, and H. Craighead, "The pull-in behavior of electrostatically actuated bistable microstructures," J. Micromech. Microeng., vol. 18, no. 5, May 2008, Art. no. 055026.

[24] J. F. Rhoads, S. W. Shaw, and K. L. Turner, "Nonlinear dynamics and its applications in micro-and nanoresonators," J. Dyn. Syst., Meas., Control, vol. 132, May 2010, Art. no. 034001.

[25] J. Yang, Y. J. Hu, and S. Kitipornchai, "Electro-dynamic behavior of an electrically actuated micro-beam: Effects of initial curvature and nonlinear deformation," Comput. Struct., vols. 96-97, pp. 25-33, Apr. 2012.

[26] S. Lenci and G. Rega, "Control of pull-in dynamics in a nonlinear thermoelastic electrically actuated microbeam," J. Micromech. Microeng., vol. 16, no. 2, pp. 390-401, Feb. 2006.

[27] R. M. C. Mestrom, R. H. B. Fey, J. T. M. van Beek, K. L. Phan, and H. Nijmeijer, "Modelling the dynamics of a MEMS resonator: Simulations and experiments," Sens. Actuators A, Phys., vol. 142, no. 1, pp. 306-315, Mar. 2008.

[28] A. M. Elshurafa, K. Khirallah, H. H. Tawfik, A. Emira, A. K. S. A. Aziz, and S. M. Sedky, "Nonlinear dynamics of spring softening and hardening in folded-MEMS comb drive resonators," J. Microelectromech. Syst., vol. 20, no. 4, pp. 943-958, Aug. 2011.

[29] S. Ilyas, F. K. Alfosail, M. L. F. Bellaredj, and M. I. Younis, "On the response of MEMS resonators under generic electrostatic loadings: Experiments and applications," Nonlinear Dyn., vol. 95, no. 3, pp. 2263-2274, Feb. 2019. 
[30] S. Ilyas, F. K. Alfosail, and M. I. Younis, "On the response of MEMS resonators under generic electrostatic loadings: Theoretical analysis," Nonlinear Dyn., vol. 97, no. 2, pp. 967-977, Jul. 2019.

[31] M. I. Younis, MEMS Linear and Nonlinear Statics and Dynamics, vol. 20. Cham, Switzerland: Springer, 2011.

[32] A. Z. Hajjaj, N. Jaber, S. Ilyas, F. K. Alfosail, and M. I. Younis, "Linear and nonlinear dynamics of micro and nano-resonators: Review of recent advances," Int. J. Non-Linear Mech., vol. 119, Mar. 2020, Art. no. 103328

[33] H. J. R. Westra, M. Poot, H. S. J. van der Zant, and W. J. Venstra, "Nonlinear modal interactions in clamped-clamped mechanical resonators," Phys. Rev. Lett., vol. 105, no. 11, Sep. 2010, Art. no. 117205.

[34] R. B. Karabalin, M. C. Cross, and M. L. Roukes, "Nonlinear dynamics and chaos in two coupled nanomechanical resonators," Phys. Rev. B, Condens. Matter, vol. 79, no. 16, Apr. 2009, Art. no. 165309.

[35] R. Potekin et al., "Multi-frequency atomic force microscopy based on enhanced internal resonance of an inner-paddled cantilever," Sens. Actuators A, Phys., vol. 273, pp. 206-220, Apr. 2018.

[36] R. M. C. Mestrom, R. H. B. Fey, K. L. Phan, and H. Nijmeijer, "Simulations and experiments of hardening and softening resonances in a clamped-clamped beam MEMS resonator," Sens. Actuators A, Phys., vol. 162, no. 2, pp. 225-234, 2010.

[37] B. Balachandran and A. H. Nayfeh, "Nonlinear motions of beam-mass structure," Nonlinear Dyn., vol. 1, no. 1, pp. 39-61, Jan. 1990.

[38] A. Erturk, J. M. Renno, and D. J. Inman, "Modeling of piezoelectric energy harvesting from an L-shaped beam-mass structure with an application to UAVs," J. Intell. Mater. Syst. Struct., vol. 20, no. 5, pp. 529-544, Mar. 2009.

[39] A. Vyas, D. Peroulis, and A. K. Bajaj, "A microresonator design based on nonlinear 1: 2 internal resonance in flexural structural modes," J. Microelectromech. Syst., vol. 18, no. 3, pp. 744-762, Jun. 2009.

[40] E. M. Lui and W.-F. Chen, "Analysis and behaviour of flexibly-jointed frames," Eng. Struct., vol. 8, no. 2, pp. 107-118, Apr. 1986.

[41] O. G. P. Baracho Neto and C. E. N. Mazzilli, "Evaluation of multi-modes for finite-element models: Systems tuned into 1: 2 internal resonance," Int. J. Solids Struct., vol. 42, nos. 21-22, pp. 5795-5820, Oct. 2005.

[42] S. Lenci and C. E. N. Mazzilli, "Asynchronous modes of vibrations in linear conservative systems: An illustrative discussion of plane framed structures," Meccanica, vol. 52, no. 13, pp. 3131-3147, Oct. 2017.

[43] C. E. Mazzilli and R. M. Brasil, "Effect of static loading on the nonlinear vibrations of a three-time redundant portal frame: Analytical and numerical studies," Nonlinear Dyn., vol. 8, no. 3, pp. 347-366, 1995.

[44] R. T. Rocha, J. M. Balthazar, A. M. Tusset, V. Piccirillo, and J. L. P. Felix, "Nonlinear piezoelectric vibration energy harvesting from a portal frame with two-to-one internal resonance," Meccanica, vol. 52, nos. 11-12, pp. 2583-2602, Sep. 2017.

[45] J. L. Palacios, J. M. Balthazar, and R. M. L. R. F. Brasil, "On non-ideal and non-linear portal frame dynamics analysis using Bogoliubov averaging method," J. Brazilian Soc. Mech. Sci., vol. 24, no. 4, pp. 257-265, Nov. 2002.

[46] R. M. L. R. F. Brasil, and C. E. N. Mazzilli, "A general FEM formulation of nonlinear dynamics applied to accessing the statical loading effect upon the dynamic response of planar frames," Appl. Mech. Rev., vol. 46, no. 11S, pp. S110-S117, Nov. 1993.

[47] A. H. Nayfeh and D. T. Mook, Nonlinear Oscillations. Hoboken, NJ, USA: Wiley, 2008.

[48] A. H. Nayfeh and B. Balakumar, Applied Nonlinear Dynamics: Analytical, Computational, and Experimental Methods. Hoboken, NJ, USA: Wiley, 2008.

[49] J. L. P. Felix, J. M. Balthazar, R. T. Rocha, A. M. Tusset, and F. C. Janzen, "On vibration mitigation and energy harvesting of a nonideal system with autoparametric vibration absorber system," Meccanica, vol. 53, no. 13, pp. 3177-3188, Oct. 2018.

[50] J. Qiu, J. H. Lang, A. H. Slocum, and A. C. Weber, "A bulkmicromachined bistable relay with U-shaped thermal actuators," J. Microelectromech. Syst., vol. 14, no. 5, pp. 1099-1109, Oct. 2005.

[51] S. A. Tella and M. I. Younis, "Multimode excitations for complex multifunctional logic device," J. Micromech. Microeng., vol. 29, no. 12, Dec. 2019, Art. no. 125017.

[52] M. Ferrari, V. Ferrari, M. Guizzetti, B. Andò, S. Baglio, and C. Trigona, "Improved energy harvesting from wideband vibrations by nonlinear piezoelectric converters," Sens. Actuators A, Phys., vol. 162, no. 2, pp. 425-431, Aug. 2010.

[53] MEMSCAP-SOIMUMPs. Accessed: Aug. 31, 2020. [Online]. Available: http://www.memscap.com/products/mumps/soimumps
[54] W. Lacarbonara, H. N. Arafat, and A. H. Nayfeh, "Non-linear interactions in imperfect beams at veering," Int. J. Non-Linear Mech., vol. 40, no. 7, pp. 987-1003, Sep. 2005.

[55] S. Ahmed, X. Zou, N. Jaber, M. I. Younis, and H. Fariborzi, "A low power micro-electromechanical resonator-based digital to analog converter," J. Microelectromech. Syst., vol. 29, no. 3, pp. 320-328, Jun. 2020.

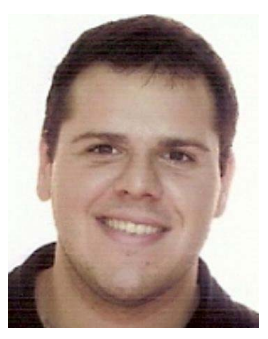

Rodrigo Tumolin Rocha was born in Brazil in 1991. He received the bachelor's degree in physics from Sao Paulo State University, Rio Claro, Brazil, in 2012, the master's degree from Sao Paulo State University, Bauru, Brazil, in 2014, and the Ph.D. degree from Sao Paulo State University, Brazil, in 2016, working on nonlinear dynamics and vibration-based energy harvesting from ambient vibration. From 2016 to 2018, he was a PostDoctoral Fellow with the Federal University of Technology - Parana (UTFPR), Ponta Grossa, Brazil, working on nonlinear dynamics, control, and energy harvesting on macro and microstructures. Since 2018, he has been a Post-Doctoral Fellow with the King Abdullah University of Science and Technology (KAUST), Thuwal, Saudi Arabia, working on modeling and experimental characterization on nonlinear dynamics of compound microstructures.

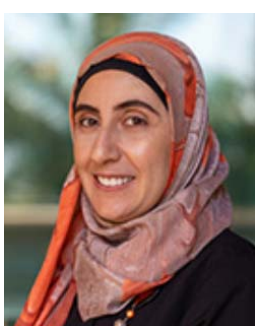

Nouha Alcheikh was born in 1984. She received the M.S. degree in electronics from the National Polytechnic Institute of Grenoble in 2007, and the $\mathrm{Ph} . \mathrm{D}$. degree in RF MEMS from Grenoble University, France, in 2011. From 2011 to 2014, she was a Post-Doctoral Fellow with the CEA-Leti/MINATEC Campus, Grenoble, France, working on force sensors and energy harvesting, and IMS, Bordeaux, France. From 2015 to 2018, she was a Post-Doctoral Fellow, working on MEMS sensors and actuators, with the King Abdullah University of Science and Technology (KAUST), Thuwal, Saudi Arabia, where she has been a Research Scientist, working on MEMS sensors and actuators, since 2018.

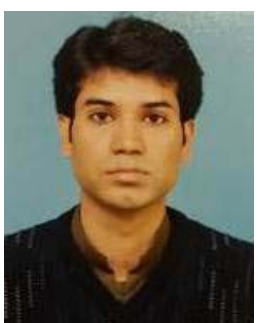

Fahimullah Khan received the B.Sc. degree in electrical engineering from the University of Engineering and Technology, Peshawar, Pakistan, in 2008, the M.S. degree in electronic engineering from the Ghulam Ishaq Khan Institute of Science and Technology, Swabi, Pakistan, in 2010, and the Ph.D. degree in micro-electronic engineering from Griffith University, Gold Coast, Australia, in December 2015. From the past few years, he was designated with various teaching and research positions. $\mathrm{He}$ is currently a Post-Doctoral Fellow with the King Abdullah University of Science and Technology. His research interests include micro/nanosystems, smart materials and structures, RF MEMS devices, and smart sensors.

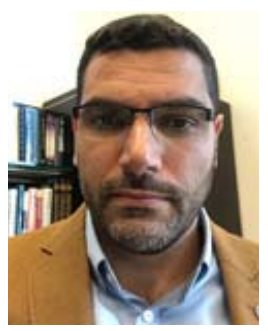

Mohammad I. Younis (Member, IEEE) received the Ph.D. degree in engineering mechanics from Virginia Polytechnic Institute and State University, Blacksburg, VA, USA, in 2004. From 2004 to 2013, he served as an Assistant Professor and then as an Associate Professor in mechanical engineering with The State University of New York (SUNY), Binghamton, NY, USA. In 2013, he moved to the King Abdullah University of Science and Technology, Saudi Arabia, where he is currently serving as a Full Professor in mechanical engineering and the Director of the MEMS and NEMS Characterization and Motion Laboratory. He holds several U.S. patents in MEMS sensors and actuators. He is a member of the American Society of Mechanical Engineers (ASME). He was a recipient of the SUNY Chancellor's Award for Excellence in Scholarship and Creative Activities in 2012, the National Science Foundation Faculty Early Career Development Award in 2009, and the Paul E. Torgersen Graduate Research Excellence Award in 2002. He serves as an Associate Editor of Nonlinear Dynamics, Journal of Computational and Nonlinear Dynamics, Journal of Vibration and Control, and Meccanica. 